\title{
Relationship Between Attention Bias and the Prognosis of Male Patients with Alcohol Dependence
}

\author{
Qingsheng Yang ${ }^{1, \dagger}$, Bohui $\mathrm{Mo}^{2, \dagger}$, Liangshuang Yin², Liangjun Pang ${ }^{3, *}$, Jiangbo Li ${ }^{4,}$ * \\ ${ }^{1}$ Hefei Center for Disease Control and Prevention, Hefei, China \\ ${ }^{2}$ Department of Psychiatry, General Hospital for Prison in Anhui Province, Hefei, China \\ ${ }^{3}$ Ward for Substance Addiction, Department of Psychiatry, Anhui Mental Health Center, Hefei, China \\ ${ }^{4}$ Department of Clinical Psychology, Wuhu Hospital Affiliated to East China Normal University, Wuhu, China \\ Email address: \\ 3295957558@qq.com (Liangjun Pang), 1015950973@qq.com (Jiangbo Li) \\ ${ }^{*}$ Corresponding author \\ $\dagger$ Qingsheng Yang and Bohui Mo are co-first authors.
}

\section{To cite this article:}

Qingsheng Yang, Bohui Mo, Liangshuang Yin, Liangjun Pang, Jiangbo Li. Relationship Between Attention Bias and the Prognosis of Male Patients with Alcohol Dependence. American Journal of Psychiatry and Neuroscience. Vol. 9, No. 3, 2021, pp. 110-114. doi: 10.11648/j.ajpn.20210903.16

Received: July 25, 2021; Accepted: August 5, 2021; Published: August 11, 2021

\begin{abstract}
Background: long term drinking in alcohol dependent patients seriously affects their physical and mental health. Abstinence treatment is very important, but there is no appropriate prediction method for the effect of abstinence in the past. Objective: In this study, we investigated word-cue attentional bias among male patients with alcohol dependence and its correlation with relapse after abstinence. Methods: Fifty male patients with alcohol dependence (ADs) and 50 male health controls (HCs) completed the Chinese version of the emotional Stroop task to assess attentional bias. The participants were evaluated at the beginning of the task using the Self-Rating Anxiety Scale (SAS) and Self-Rating Depression Scale (SDS). Results: The reaction times of ADs for neutral, negative, and alcohol-related words were significantly higher than those of HCs. The error index of ADs for neutral and negative words is worse than that of HCs. The period of maintained abstinence among ADs was positively correlated with the reaction time to negative words and the error index for negative and alcohol-related words. Linear regression showed the error index of alcohol-related words to be an import factor in terms of relapse. Conclusion: The number of errors in alcohol-related attentional bias cue words may be a predictor of the effect of abstinence.
\end{abstract}

Keywords: Alcohol Drinking, Alcoholism, Attentional Bias, Stroop Test

\section{Introduction}

Alcohol abuse leads to a series of serious health and social problems. Research has indicated that an estimated $4.9 \%$ of the world's adult population (240 million people) suffers from alcohol use disorder ( $7.8 \%$ of men and $1.5 \%$ of women), with alcohol causing an estimated 257 disability-adjusted life years lost per 100,000 population [1]. Studies have revealed long-standing heavy alcohol abuse leads to disproportionate loss of cerebral white matter and impairments in executive function [2]. These lesions ultimately lead to the cognitive decline.

Janssen et al. [3] they found that certain measures of alcohol-related attentional bias predicted later alcohol use in young adolescents. Excessive drinking sensitizes alcohol abusers' attentional responsiveness to alcohol-related stimuli $[4,5]$. Increasing evidence has shown that $\mathrm{AD}$ have significant attentional bias to alcohol [6-10] and that attentional bias may play a crucial role in the formation of $A D$ and the return to drinking after abstinence. Even though AD has been the focus of multiple studies, there are many aspects of the condition, such as the relationship between attention bias and abstinence of $\mathrm{AD}$, that remain unclear. The purpose of this study was to explore the correlation between attention bias and the prognosis of $\mathrm{AD}$. 


\section{Materials and Methods}

\subsection{Grouping}

This study included 50 male AD and 50 healthy controls. $\mathrm{AD}$ were recruited from Anhui Mental Health Center. The inclusion criteria were as follows: (1) patients satisfied the diagnostic criteria for $\mathrm{AD}$ specified in the International Statistical Classification of Diseases and Related Health Problems 10th Revision (ICD-10); (2) treatment time of hospitalization was within 5-15 days without withdrawal symptoms following the experiment (AD hospitalized for abstinence); and (3) the mini-mental state examination (MMSE) score was larger than 26 points. The exclusion criteria were as follows: (1) any organic brain disease shown on computed tomography (CT) scans; (2) neuropsychiatric disorders or other diseases that may cause cognitive impairment; or (3) drug dependence history (except tobacco). 50 healthy men were recruited as controls from the community in Hefei, China. Patient demographics are presented in Table 1. All participants were right-handed. This study was approved by the Ethical Committee of Hefei Center for Disease Control and Prevention and Anhui Mental Health Center. All procedures were conducted in accordance with the guidelines put forth in the Declaration of Helsinki, and written informed consent was obtained from all participants.

\subsection{Test Methods}

\subsubsection{Assessment Tools}

The assessment tools included the (1) Mini-Mental State Examination (MMSE); (2) Self-Rating Anxiety Scale (SAS); (3) Self-Rating Depression Scale (SDS); (4) Michigan Alcohol Use Questionnaire (MAST); and (5) telephone interviews. All the discharged AD were contacted monthly to record the time of abstinence after quitting the use of alcohol.

\subsubsection{Attention Bias Test}

We used the Stroop paradigm to present participants with Chinese words in four different colours. Numbers 1-4 were used to represent red, yellow, blue, and green, respectively. The test was divided into two stages with 30 words presented in each stage, including neutral words (i.e., desk, cabinet, wooden bed), negative words (i.e., death, funeral, loneliness), and clue words related to alcohol (i.e., good wine, beer, rice wine). The three types of words were alternately and randomly displayed on a computer screen. All tests were conducted in a quiet environment. Participants were seated $50 \mathrm{~cm}$ from the screen and asked to press the number key corresponding to the colour of the characters immediately after seeing the stimulus word. The computer automatically recorded the reaction time and the number of mistakes each participant made.

\subsection{Statistical Analysis}

All data were analysed using SPSS 13.0 (SPSS Inc., Chicago, USA). Differences and correlations between groups were analysed using the student t-test and chi-square test. The Pearson correlation coefficient was used to evaluate the extent to which variables were linearly related. P-values of less than 0.05 were considered statistically significant.

\section{Results}

\subsection{Demographics and Alcohol Consumption Data of AD and HC Groups}

As shown in Table 1, the $\mathrm{AD}$ and control groups did not differ significantly in age $(\mathrm{t}=5.542, \mathrm{P}=0.589)$, years of education $(\mathrm{t}=1.537, \mathrm{P}=0.127)$, marital status $\left(\mathrm{X}^{2}=4.016\right.$ $\mathrm{P}=0.134)$, professional situation $\left(\mathrm{X}^{2}=0.421, \mathrm{P}=0.517\right)$, or family history of alcohol consumption $\left(\mathrm{X}^{2}=0.543, \mathrm{P}=0.461\right)$. In the $\mathrm{AD}$ group, the age of starting drinking, duration of alcohol consumption (years), age of addiction, duration of addiction (years), and duration of abstinence (months) were shown in Table 1. The AD abstinence time of this group was 2-8 months.

Table 1. Demographics and history of alcohol.

\begin{tabular}{lll}
\hline & AD group & Control group \\
\cline { 2 - 3 } & $\mathbf{( n = 5 0 )}$ & $\mathbf{( n = 5 0 )}$ \\
\hline Age (year) & $41.14 \pm 6.37$ & $40.46 \pm 6.17$ \\
Years of education (year) & $10.70 \pm 2.50$ & $10.02 \pm 1.88$ \\
Marital status & & \\
Married & 36 & 44 \\
Unmarried & 12 & 5 \\
Divorced & 2 & 1 \\
Profession & & \\
Mental work & 14 & 17 \\
Manual labor & 36 & 33 \\
Family history of drinking & 5 & 3 \\
Start drinking age & $20.74 \pm 5.11$ & - \\
Alcohol duration (year) & $16.26 \pm 6.46$ & - \\
Addiction age & $34.42 \pm 5.04$ & - \\
Addiction Duration (year) & $6.52 \pm 4.75$ & - \\
Abstinence Time (month) & $5.36 \pm 3.06$ & - \\
\hline
\end{tabular}

\subsection{Comparison of Psychology Scale Scores}

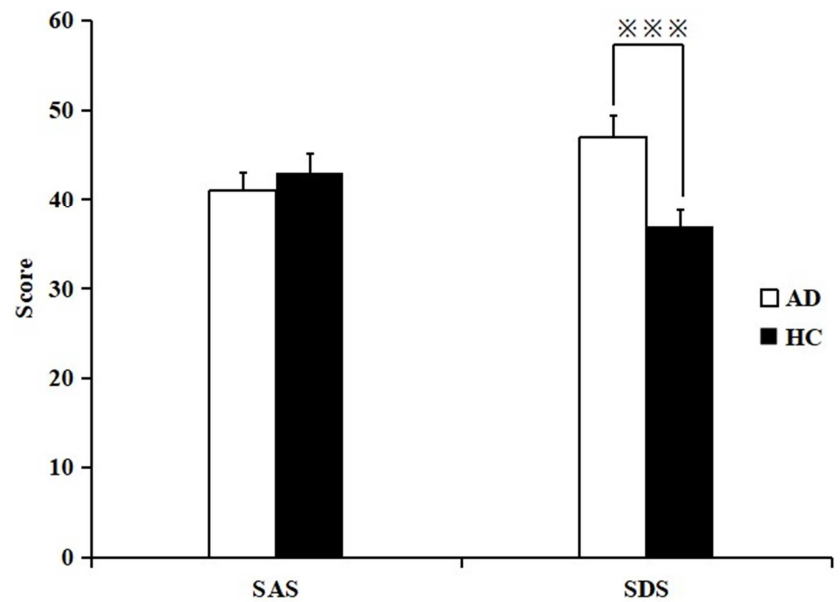

Figure 1. Data are expressed as means \pm standard error of the mean (SEM), $※ ※ \times 0.001$ Attention bias comparison.

As shown in Figure 1, SDS scores in the AD group were significantly higher than those in the HC group (47.25 \pm 12.84 vs. $38.37 \pm 5.78, \mathrm{P}<0.001)$. The SAS scores did not differ 
significantly between the $\mathrm{AD}$ and $\mathrm{HC}$ groups. The mean MAST score in the AD group was 21.86 \pm 7.72 .

As shown in Figures 2 and 3, the response time for neutral, negative, and cue words differed significantly between the AD sand $\mathrm{HC}$ groups $(\mathrm{P}<0.001)$. The error indexes for neutral and negative words also differed significantly between the two groups (all $\mathrm{P} \leq 0.001$ ). However, the error index for cue words did not differ significantly.

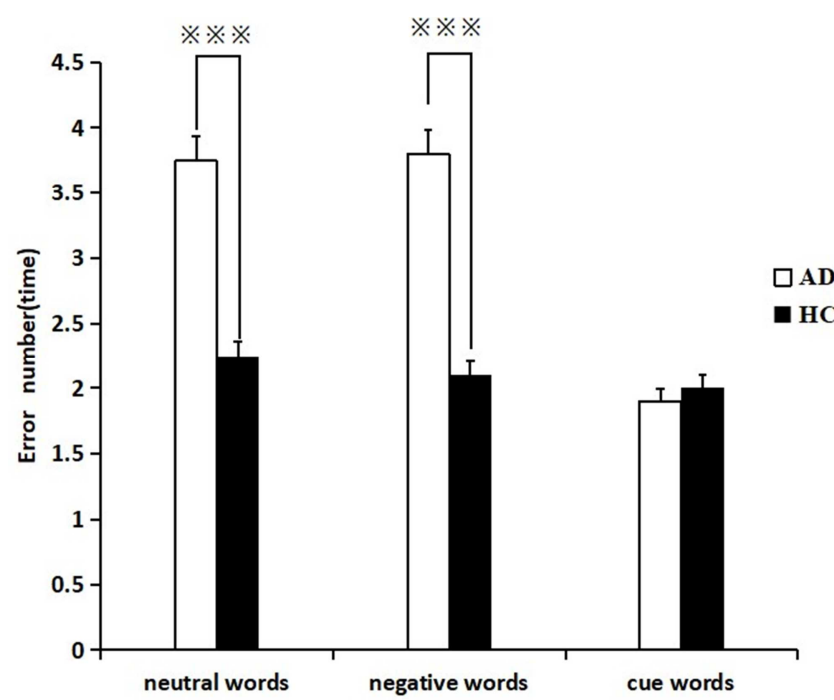

Figure 2. Data are expressed as means \pm standard error of the mean (SEM), $※ ※ \times 0.001$.

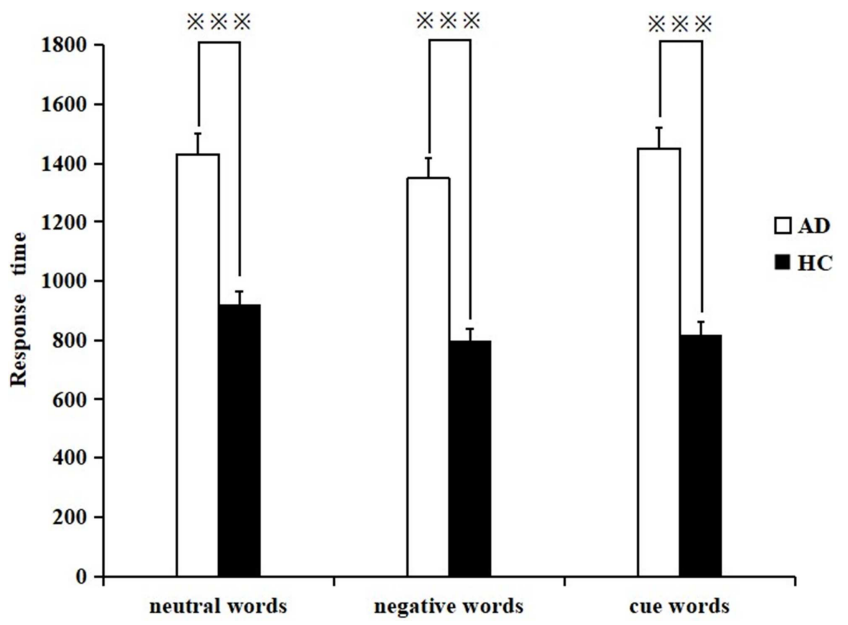

Figure 3. Data are expressed as means \pm standard error of the mean (SEM), $※ ※ ※<0.001$.

\subsection{Correlation Between Abstinence Time After Hospital Discharge and Attention Bias}

As shown in Table 2, the time of abstinence of $\mathrm{AD}$ after hospital discharge was positively correlated with the response time for negative words. The time of abstinence after hospital discharge was also positively correlated with the error indexes for negative and cue words.

Table 2. Correlation between the abstinence time of AD after hospital discharge and attention bias.

\begin{tabular}{|c|c|c|c|c|c|c|}
\hline \multirow{2}{*}{ Spearman coefficient } & \multicolumn{2}{|l|}{ neutral words } & \multicolumn{2}{|l|}{ negative words } & \multicolumn{2}{|l|}{ cue words } \\
\hline & response time & error number & response time & error number & response time & error number \\
\hline$r$ value & 0.069 & 0.268 & 0.408 & 0.431 & 0.244 & 0.563 \\
\hline$P$ value & 0.635 & 0.160 & 0.003 & 0.002 & 0.087 & 0.000 \\
\hline
\end{tabular}

\subsection{Correlation Between Duration of Abstinence After Discharge and Drinking Behavior and Mood}

As shown in Table 3, the withdrawal time of AD after discharge had no significant correlation with the age of starting drinking, duration of drinking, age of addiction, duration of addiction, SAS, SDS, and MAST.

Table 3. Correlation between duration of abstinence and drinking behavior and mood after abstinence treatment.

\begin{tabular}{|c|c|c|c|c|c|c|c|}
\hline Spearman Coefficient & Start drinking age & Alcohol duration & Addiction age & Addiction Duration & SAS & SDS & MAST \\
\hline$r$ value & -0.190 & 0.150 & -0.198 & 0.092 & -0.077 & -0.078 & 0.009 \\
\hline$P$ value & 0.187 & 0.299 & 0.169 & 0.525 & 0.595 & 0.591 & 0.950 \\
\hline
\end{tabular}

\subsection{Multivariate Regression Analysis of the Influencing Factors of Attentional Bias of Alcohol Cue Words in AD Group}

In order to further explore the effect of each influencing factor on attentional bias, the time of continuous abstinence after discharge was taken as the dependent variable, the reaction time of negative words and the number of errors between negative words and cue words were taken as the independent variables for multivariate regression analysis $(\mathrm{PIN}=0.05$, POUT $=0.1$ ). Only the number of errors in cue words was selected (table 4).

Table 4. Multivariate regression analysis of attention bias in AD.

\begin{tabular}{|c|c|c|c|c|c|}
\hline Variable & Regression coefficients (B) & Standard error (SE) & Standard regression $(\beta)$ & $T$ value & $P$ value \\
\hline response time of negative words & 0.005 & 0.002 & 0.481 & 1.840 & 0.073 \\
\hline the error number of negative words & 0.181 & 0.217 & 0.134 & 0.831 & 0.411 \\
\hline the error number of cue words & 0.848 & 0.262 & 0.420 & 3.233 & 0.002 \\
\hline
\end{tabular}




\section{Discussion}

$\mathrm{AD}$ introduces a series of social problems and threatens public health. In recent years, studies on the relationship between attentional bias and drinking in $\mathrm{AD}$ has attracted extensive attention. Fadardi et al. [4] found that the dependent drinkers have greater alcohol attentional bias, and excessive drinking sensitizes alcohol abusers' attentional responsiveness to alcohol-related stimuli. Furthermore, Field et al. [11] found that Among young individuals who were light drinkers, attention bias was positively associated with the subjective desire for alcohol and weekly alcohol consumption. Adams et al. [12] Studies have shown that participants who received a moderate dose of alcohol $(0.40 \mathrm{~g} / \mathrm{kg})$ were faster to respond to alcohol-related stimuli compared to participants receiving a low dose of alcohol or placebo. It is necessary to study the prognosis of alcohol-dependent patients and to improve their attentional bias to alcohol $[13,14]$.

We demonstrated that the response times for neutral, negative, and cue words in the AD group were significantly prolonged. These results indicate slow to respond or executive dysfunction in $\mathrm{AD}$, This is consistent with previous studies [2]. We also found significant differences in the error indexes for neutral and negative words between the two groups, but not in the error index for cue words. This indicates that $\mathrm{AD}$ can focus their attention on the alcohol-related information and reduce the number of experimental errors. Less attention is paid to other aspects; therefore, the number of errors is significantly higher. Excessive attention to alcohol-related information and lack of attention to other aspects may be an important psychological mechanism for AD difficulties in abstinence and reduced social function. In alcohol dependents with depression, a memory bias for alcohol-related material was found, suggesting that this group may be more pre-occupied with alcohol than patients without such co-morbidity [15]. Our study showed higher degree of depression in the AD group than in the $\mathrm{HC}$ group, which indicates that depressed mood is common among $\mathrm{AD}$, and depressed mood may be a important factors for continued drinking. However, there was no significant correlation between the time of abstinence after hospital discharge and degree of depression in our study, the effect of depressed mood on after hospital repeat drinking may be uncertain.

$\mathrm{AD}$ has obvious attentional bias to alcohol, and poor attention to things other than alcohol, showing sluggish reaction and poor executive function. However, it has not been reported that the degree of attentional bias to alcohol can predict the time to alcohol withdrawal in AD. We want to find out the relationship between attentional bias and the duration of abstinence, and whether attentional bias can be used to predict the duration of abstinence, We found that the abstinence duration of $\mathrm{AD}$ after discharge was not significantly correlated with the age of starting drinking, duration of drinking, age of addiction, duration of addiction, anxiety, depression, and MAST scores, which we were pleasantly surprised to find that the time of abstinence after hospital discharge was positively correlated with the response time for negative words and the error indexes for negative words and cue words. This shows the less attention paid to negative information and alcohol clue words, the longer the abstinence time. Our results of Multiple regression analysis suggest that among the indicators of attentional bias, only the number of errors in alcohol cue words is an important factor affecting the duration of abstinence. The number of errors in alcohol cue words may be an important indicator for predicting the duration of abstinence.

In conclusion, male $\mathrm{AD}$ patients do have attention bias towards alcohol, which this characteristic may be related to the impairment of executive function, excessive dependence on alcohol and difficulty in abstinence, therefore It may also related to the occurrence, development and prognosis of $\mathrm{AD}$, and the index of attention bias may be used as a predictor of $\mathrm{AD}$ prognosis. Our findings may provide a theoretical basis for the development of treatment methods for abstinence; Establish a training system to reduce AD's focus on alcohol, increase the time of physical activity training and social interaction not related to alcohol, and increase education regarding the negative effects of long-term drinking on the body. During the training, the attention bias and the number of errors in alcohol cues can be checked to test the effect of the training, the training techniques of reduce attentional bias to alcohol and prolong the duration of abstinence, as well as the application of attentional bias techniques to predict the duration of abstinence are worthy of further study.

\section{Conflict of Interest}

There is no conflict of interest between the authors.

\section{Research Ethics}

This study was approved by the Ethical Committee of Hefei Center for Disease Control and Prevention and Anhui Mental Health Center. All procedures were conducted in accordance with the guidelines put forth in the Declaration of Helsinki, and written informed consent was obtained from all participants.

\section{Funding}

This research did not receive any specific grant from funding agencies in the public, commercial, or not-for-profit sectors.

\section{References}

[1] Gowing LR, Ali RL, Allsop S, Marsden J, Turf EE, West R, Witton J. Global statistics on addictive behaviours: 2014 status report. Addiction 2015; 110: 904-19.

[2] Suzanne M de la Monte, Jillian J Kril. Human alcohol-related neuropathology. Acta Neuropathol 2014; 127 (1), 71-90. 
[3] Janssen $\mathrm{T}$, Larsen $\mathrm{H}$, Vollebergh WAM, Wiers RW. Longitudinal relations between cognitive bias and adolescent alcohol use. Addict Behav 2015; 44, 51-7.

[4] Fadardi JS, Cox WM. Alcohol attentional bias: drinking salience or cognitive impairment? Psychopharmacology 2006; 185: 169-78.

[5] Cox WM, Hogan LM, Kristian MR, Race JH. Alcohol attentional bias as a predictor of alcohol abusers' treatment outcome. Drug Alcohol Depend 2002; 68: 237-43.

[6] Julia M A Sinclair, Matthew Garner, Sonja C Pasche, Thomas B Wood, David S Baldwin. Attentional biases in patients with alcohol dependence: influence of coexisting psychopathology, Hum Psychopharmacol 2016; 31 (6): 395-401.

[7] Mélanie Brion, Valérie Dormal, Séverine Lannoy, Serge Mertens, Philippe de Timary, Pierre Maurage. Imbalance between cognitive systems in alcohol-dependence and Korsak off syndrome: An exploration using the Alcohol Flanker Task, J Clin Exp Neuropsychol 2018; 40 (8): 820-31.

[8] Annie Roy-Charland, Andréanne Plamondon, Andrew S Homeniuk, Corie Ann Flesch, Raymond M Klein, Sherry H Stewart. Attentional bias toward alcohol-related stimuli in heavy drinkers: evidence from dynamic eye movement recording, Am J Drug Alcohol Abuse 2017; 43 (3): 332-40.

[9] Zoé Bollen, Nicolas Masson, Samuel Salvaggio, Fabien D'Hondt, Pierre Maurage Craving is everything: An eye-tracking exploration of attentional bias in binge drinking, $J$ Psychopharmacol 2020; 34 (6): 636-47.
[10] Charlotte R Pennington, Daniel J Shaw, Jennifer Adams, Phoebe Kavanagh, Holly Reed, Madeleine Robinson, Emily Shave, Hollie White. Where's the wine? Heavy social drinkers show attentional bias towards alcohol in a visual conjunction search task. Addiction 2020; 115 (9): 1650-9.

[11] Field M, Christiansen P, Cole J, Goudie A. Delay discounting and the alcohol Stroop in heavy drinking adolescents. Addiction 2007; 102: 579-86.

[12] Adams S, Ataya AF, Attwood AS, Munafò MR. Effects of acute alcohol consumption on alcohol-related cognitive biases in light and heavy drinkers are task-dependent. $J$ Psychopharmacol 2012; 26: 245-53.

[13] Timo L Kvamme, Mads U Pedersen, Morten Overgaard, Kristine Rømer Thomsen, Valerie Voon. Pilot study: Improving attention bias modification of alcohol cues through concealed gaze-contingent feedback in alcohol dependence. Addict Behav Rep 2019, Published online 2019 Nov 6. doi: 10.1016/j.abrep. 2019.100231

[14] Carmen Díaz-Batanero, Sara Domínguez-Salas, Enrique Moraleda, Fermín Fernández -Calderón, Oscar M Lozano. Attentional bias toward alcohol stimuli as a predictor of treatment retention in cocaine dependence and alcohol user patients. Drug Alcohol Depend 2018Jan1; 182: 40-7.

[15] Fridrici C, Driessen M, Wingenfeld K, Georg K, Kissler J, Beblo $\mathrm{T}$. Investigating biases of attention and memory for alcohol-related and negative words in alcohol-dependents with and without major depression after day-clinic treatment. Psychiatry Res 2014; 218: 311-8. 